\title{
Modifications in ankle dorsiflexor activation by applying a torque perturbation during walking in persons post-stroke: a case series
}

\author{
Andreanne K Blanchette ${ }^{1,2,3}$, Martin Noël ${ }^{2}$, Carol L Richards ${ }^{1,2,3}$, Sylvie Nadeau ${ }^{1,4,5}$ and Laurent J Bouyer ${ }^{1,2,3^{*}}$
}

\begin{abstract}
Background: Results obtained in a previous study (Gait Posture 34:358-363, 2011) have shown that, in non-disabled participants, a specific increase in ankle dorsiflexor (Tibialis anterior [TA]) activation can be induced by walking with a torque perturbation that plantarflexes the ankle during the swing phase. After perturbation removal, the increased TA activation persisted temporarily and was associated with a more dorsiflexed ankle during swing. The objective of the present case-series study was to verify if these results can be reproduced in persons post-stroke.

Methods: Six participants who sustained a stroke walked on a treadmill before, during and after exposure to a torque perturbation applied at the ankle by a robotized ankle-foot orthosis. Spatiotemporal gait parameters, ankle and knee kinematics, and the electromyographic activity of TA and Soleus were recorded. Mean amplitude of the TA burst located around toe off and peak ankle dorsiflexion angle during swing were compared across the 3 walking periods for each participant.

Results: At the end of the walking period with the perturbation, TA mean amplitude was significantly increased in 4 of the 6 participants. Among these 4 participants, modifications in TA activation persisted after perturbation removal in 3 of them, and led to a statistically significant increase in peak dorsiflexion during swing.

Clinical implications: This approach may be helpful to evaluate the residual adaptive capacity in the ankle dorsiflexors after a stroke and guide decision-making for the selection of optimal rehabilitation interventions. Future work will investigate the clinical impact of a multiple-session gait training based on this approach in persons presenting a reduced ankle dorsiflexion during the swing phase of walking.
\end{abstract}

Keywords: Human locomotion, Motor control, Ankle dorsiflexors, Robotic device, Stroke, Adaptation, Rehabilitation

\section{Background}

After a central nervous system lesion (CNS), adaptation of the walking pattern to meet varied environmental demands, such as walking on a sandy beach or in snow, becomes more difficult. The neural control of walking involves complex interactions between different levels of commands. Central commands, from voluntary and automatic drive, predict the muscle activation pattern needed to perform the movement before its execution (feedforward control), while peripheral sensory inputs are involved in correction

\footnotetext{
* Correspondence: laurent.bouyer@rea.ulaval.ca

'Multidisciplinary Team in Locomotor Rehabilitation, Canadian Institutes of Health Research, Quebec, Canada

${ }^{2}$ Center for Interdisciplinary Research in Rehabilitation and Social Integration, 525 Wilfrid-Hamel Blvd, Quebec City G1M 2S8, Canada

Full list of author information is available at the end of the article
}

during movement execution (feedback control) and also in providing error feedback to prepare the next movement [1]. The CNS is constantly adjusting locomotor commands to the environmental constraints. A contribution of the cerebellum [2] and of the corticospinal tract [3,4] in locomotor adaptation have been proposed in the literature.

Over the last 20 years, paradigms consisting of walking in an experimentally controlled perturbing environment have been developed to evaluate this locomotor adaptive capacity [5-20]. A perturbation that induces a movement error during walking triggers a recalibration of central commands to adapt the muscle activation pattern to its presence. After perturbation removal, this recalibration persists temporarily and can lead to changes in lower limb kinematics (e.g. [10,11,13-15,17,19]). The perturbations

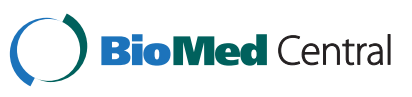


used in these studies can be separated into 2 types: one that primarily affects a single lower limb (e.g. adding a weight on the leg or resisting a movement with a robotic device); and another that uses the walking surface to perturb interlimb coordination (e.g. split-belt treadmill).

A few recent studies have demonstrated that persons post-stroke are still able to adapt spatiotemporal gait parameters, such as step length, double support or singlelimb support time to the presence of a perturbation during walking [21-24]. Adaptation of these parameters likely results from coordinated modifications in the activation of several muscle groups. To date, no experimental protocols have evaluated the residual adaptive capacity of a specific muscle group within a single limb while walking in a perturbing environment. This approach may be helpful to guide decision-making for the selection of optimal rehabilitation interventions in situations where the walking deficit is more localized.

A robotized ankle-foot orthosis that uses a hybrid drive system (electrohydraulic) was developed in our laboratory to apply phase-specific torque perturbations at the ankle joint during walking [25]. The effects of walking with a perturbation that targets the ankle joint during the swing phase on the locomotor pattern of non-disabled subjects were quantified in a previous study [19]. The purpose of the present case series was to evaluate ankle dorsiflexor residual adaptive capacity when persons post-stroke are exposed to a similar perturbation. Based on the results obtained in non-disabled subjects [19], we hypothesized that walking with a perturbation that plantarflexes the ankle during the swing phase would induce an increase in tibialis anterior (TA) activation in these subjects. We also hypothesized that such modifications would carry over after perturbation removal and lead to an increase in ankle dorsiflexion during the swing phase of walking. Preliminary results were published in abstract form [26].

\section{Methods}

Participants

Six individuals (S1 - S6) were recruited from the community to participate to the present experiment (Table 1).
Inclusion criteria were as follows: participants had sustained a stroke more than 6 months prior to the study (chronic lesion), were discharged from rehabilitation, and were able to walk overground for at least 10 meters independently (i.e. without human assistance). Subjects were excluded if they had unstable health conditions or cardiovascular, pulmonary and/or cognitive deficits that could affect performance during the experiment. To reduce potential skin abrasions from the orthosis, persons having no light touch sensation on the foot and around the ankle were also excluded.

\section{General protocol}

For the Evaluation of ankle dorsiflexor adaptive capacity (see below), the test paradigm consisted of walking on a treadmill before, during and after exposure to a specific perturbation applied at the ankle. Before testing, each subject underwent a Clinical examination performed by a physical therapist to characterize ankle motor impairments. This protocol was approved by the Quebec City Physical Rehabilitation Institute human research review board and all subjects provided written informed consent prior to their entry in the study.

\section{Clinical examination}

The Clinical examination documented the presence of motor impairments affecting the paretic ankle joint, such as dorsiflexor paresis, plantarflexor hypertonicity, or contractures $[27,28]$. Maximal passive ankle dorsiflexion was measured with a manual goniometer, with the participant laying supine with knee and hip joints at 0 degrees of flexion. Plantarflexor contractures were considered as present if passive dorsiflexion was less than or equal to 10 degrees [29]. Plantarflexor hypertonicity was evaluated with the Modified Ashworth Scale (MAS; [30]), which assesses ankle resistance to a velocity dependent stretch reflex using a 5point nominal scale (ranging from $0=$ "No increase in muscle tone" to 4 = "Affected part(s) rigid in flexion or extension"). Voluntary dorsiflexor strength was assessed using Daniels and Worthingham's manual muscle testing grades (MMT; [31]), ranging from $5=$ "Completes full range and

Table 1 Participant characteristics

\begin{tabular}{|c|c|c|c|c|c|c|c|c|}
\hline Participant & $\begin{array}{l}\text { Gender, } \\
\text { M/F }\end{array}$ & $\begin{array}{l}\text { Age, } \\
\text { year }\end{array}$ & Height, cm & Weight, kg & Lesion & $\begin{array}{l}\text { Time post-lesion, } \\
\text { month }\end{array}$ & $\begin{array}{c}\text { Paretic side, } \\
\text { R/L }\end{array}$ & Walking aid \\
\hline S1 & M & 69 & 168 & 82 & Left ischemic stroke (frontoparietal) & 61 & $\mathrm{R}$ & Cane + AFO \\
\hline $\mathrm{S} 2$ & $F$ & 52 & 168 & 67 & Left ischemic stroke (frontoparietal) & 235 & $\mathrm{R}$ & None \\
\hline S3 & M & 70 & 168 & 70 & Right ischemic stroke (frontoparietal) & 27 & $L$ & Cane \\
\hline S4 & M & 56 & 168 & 109 & Right ischemic stroke (medulla oblongata) & 42 & $L$ & Cane \\
\hline S5 & M & 74 & 174 & 70 & Left ischemic stroke (pons) & 30 & $\mathrm{R}$ & None \\
\hline S6 & M & 79 & 170 & 68 & Right ischemic stroke (frontal) & 29 & $L$ & None \\
\hline
\end{tabular}

Participant demographic (gender, age, weight, height) and lesion characteristics (location, time post-lesion, paretic side, walking aid) are described. F: female; M: male; R: right; L: left; AFO: Ankle-foot orthosis. 
holds against maximal resistance" to $0=$ "No palpable contraction".

\section{Evaluation of ankle dorsiflexor adaptive capacity}

Participants walked with an electrohydraulic ankle-foot orthosis (EHO; [25]) on a treadmill at comfortable speed (range: $1.5-2.5 \mathrm{~km} / \mathrm{h}$ ) for 3 periods (duration ranging between 1 and 3 minutes): before, during and after walking with a perturbation. The CONTROL walking period was used to characterize individual baseline walking patterns (approximately 50 strides). It was followed by the PERTURBATION walking period, during which a torque perturbation was applied at the paretic ankle on every stride of walking. Participants were asked to: "Resist the force and try to walk normally". The number of strides with a stable perturbation application was approximately 50. Modifications induced by walking with the perturbation were studied during this period. The POST walking period was used to document aftereffects following perturbation removal (approximately 50 strides). Participants walked continuously with the robotized orthosis during the 3 walking periods, looking at a target located $3.5 \mathrm{~m}$ straight ahead. When no perturbation was applied, the EHO actively compensated for friction and energy loss across the hydraulic circuit in order to minimize its effects on the subject's natural walking pattern ("active torque cancellation mode"; [25]). For security reasons, participants wore a harness attached to a ceiling-mounted suspension system (no weight support was provided) and lightly held the front handlebar of the treadmill. No walking aids (cane or ankle-foot orthosis) were used during the experiment. They were not informed of the exact time of transitions between walking periods to avoid anticipatory adjustments prior to these transitions.

The perturbation was applied using a custom-designed EHO that uses a hybrid drive system combining the advantages of pneumatic, hydraulic, and electric systems (Figure 1; [25]). The EHO is characterized by lightweight actuators (pneumatic cylinders), a simplified force control algorithm (electric motor with PID controller), high power, and a short time constant (energy transfer by hydraulic fluid). The hybrid system is composed of an electric motor coupled to a hydraulic closed-loop system. The electric motor actuates a master cylinder (remote from the subject) that then transfers the mechanical power through hoses to a slave cylinder attached to a customdesigned aluminum ankle-foot orthosis worn by the participant. To optimize performance, pneumatic cylinders and hoses are filled with water instead of air to minimize compressibility and to improve system performance at higher frequencies.

The EHO enables the application of selected torque perturbations at the ankle joint at targeted moments of the gait cycle by rapidly switching from torque cancellation to torque generation. The torque generation mode is controlled in real time using: 1) a force

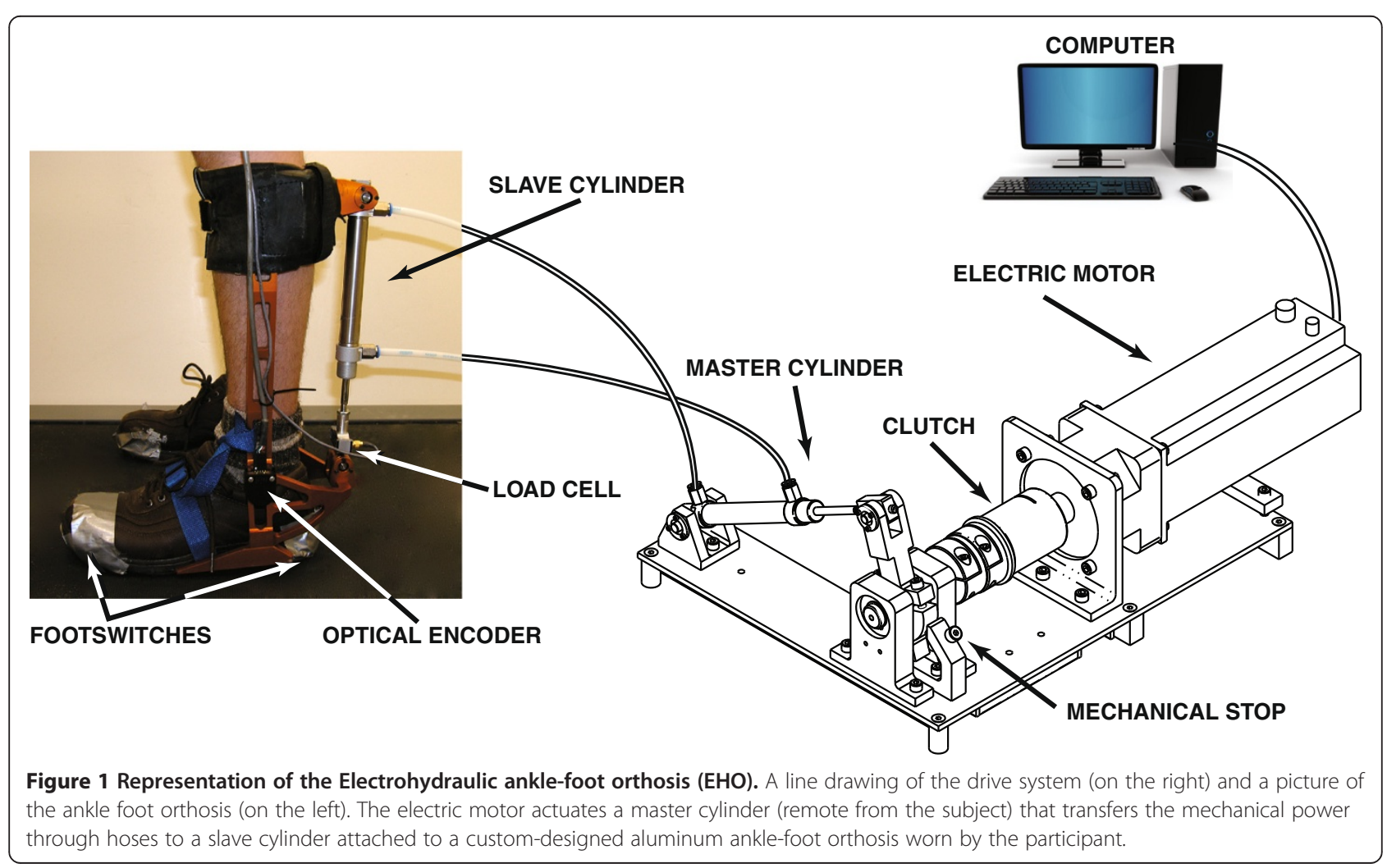


signal from a load cell (Transducer Techniques Inc.) located at the lower extremity of the slave cylinder; 2) ankle angular position signals from an optical encoder (US Digital Inc.) located on the joint of the orthosis; and 3) signals from a footswitch placed under the heel as an indication of initial foot contact with the ground. More details can be found in Noël et al. [25].

In the present study, the perturbation consisted in applying a parabolic shape torque in the plantarflexion direction during the swing phase of walking, where the ankle has to move towards dorsiflexion in order to clear the toes off the ground. The exact timing and duration of the perturbation were adjusted for each participant on the basis of the ankle movements recorded during the baseline walking period. The perturbation was applied during the first half of swing phase (initial to mid-swing) to avoid disturbances in the preparation of foot contact with the ground. Perturbation onset was set approximately $50 \mathrm{~ms}$ after the initiation of swing phase (toe off) and the duration of the perturbation was fixed between 150 and $250 \mathrm{~ms}$.

The following mathematical equation was used:

$$
\tau=K\left(-4 u^{2}+4 u\right)
$$

In this equation, $\mathrm{u}=\mathrm{t} / \mathrm{T}$ where $\mathrm{t}=$ the onset time, and $\mathrm{T}=$ the total duration of the torque perturbation (seconds). $\mathrm{K}$ corresponds to the targeted peak amplitude $(\mathrm{Nm})$. K was adjusted on an individual basis such that the smallest torque that induced a visible plantarflexion deviation in ankle trajectory was used.

Spatiotemporal gait parameters, ankle and knee kinematics and EMG data were recorded during this evaluation. Custom-made footswitches placed under the heel and big toe of both shoes were used to characterize spatiotemporal gait parameters. EMG activity of the tibialis anterior (TA) and Soleus (SOL) muscles of the paretic lower limb was recorded using disposable surface electrodes (KENDALL MEDI-TRACE 200, Covidien, Mansfield, USA) and a custom-made EMG amplifier. Electrodes were placed according to the Surface ElectroMyoGraphy for the Non-Invasive Assessment of Muscles (SENIAM) recommendations [32]. EMG signals were band-pass filtered $(10-800 \mathrm{~Hz})$ prior to acquisition, and then digitally filtered (zero-lag Butterworth 4th order filter; band-pass 10-450 Hz) before rectification. Knee and ankle angular displacements were measured with an electrogoniometer (Biometrics Ltd., Ladysmith, USA), and an optical encoder placed on the EHO (US Digital Inc., Washington, USA), respectively. Force applied by the slave cylinder was measured using a load cell (Transducer techniques, Temecula, USA). All signals were digitized at $1000 \mathrm{~Hz}$.

\section{Data analysis}

Custom-made software written in MATLAB (The MathWorks Inc., Natick, USA) was used for data analysis. Data recorded during the Evaluation of ankle dorsiflexor adaptive capacity were separated into individual strides based on footswitch signals, timenormalized to $100 \%$ for each stride, and then divided into 50 bins of equal width ( $2 \%$ of the gait cycle). Mean amplitude for each of these bins was reported for ankle and knee kinematics, torque, and EMG data.

To characterize the adaptive capacity of ankle dorsiflexors, TA mean amplitude of the burst located around toe off was calculated. A visual inspection of the mean TA activation profile in the presence of the perturbation enabled the identification of the TA burst location (around toe off) normally considered to be a concentric EMG activity burst involved in the dorsiflexion movement during swing. SOL mean amplitude was also measured in this zone to document potential coactivation when walking with the perturbation. Torque applied at the ankle was obtained by multiplying the linear force measured with the load cell by the length of the effective orthosis lever arm [25]. For each subject, the torque measured at baseline was subtracted from the torque recorded during the PERTURBATION walking period. Amplitude and position of peak added torque were then identified.

\section{Statistics}

To evaluate ankle dorsiflexor adaptive capacity, the following 3 epochs were defined:

(a) "Baseline": mean of the last 5 strides of the CONTROL period;

(b) "Adapted state": mean of the last 5 strides of the PERTURBATION period;

(c) "Aftereffects": mean of the first 5 strides of the POST period;

For each participant, one-Way ANOVAs were first used to determine if walking with the perturbation was having an overall effect on TA mean amplitude and maximal dorsiflexion during swing (intra-subject comparisons). T-tests were then used with Bonferroni correction to identify which epochs were different from baseline. Level of significance was set at 0.05.

\section{Results}

Ankle motor impairments in persons post-stroke

As demonstrated by the clinical assessment, persons post-stroke recruited in this study had varying levels of ankle motor impairments (Table 2). 
Table 2 Clinical measurements characterizing ankle motor impairments (paretic ankle)

\begin{tabular}{cccc}
\hline \multicolumn{4}{c}{ CLINICAL MEASUREMENTS } \\
\hline Participant & $\begin{array}{c}\text { Passive } \\
\text { dorsiflexion } \\
\text { angle, degree }\end{array}$ & $\begin{array}{c}\text { Plantarflexor } \\
\text { hypertonicity, } \\
\text { MAS score (0-4) }\end{array}$ & $\begin{array}{c}\text { Dorsiflexor strength, } \\
\text { MMT grade (0-5) }\end{array}$ \\
\hline S1 & -10 & 4 & 1 \\
S2 & -2 & 3 & 2 \\
S3 & 7 & $1+$ & 2 \\
S4 & 18 & 0 & 4 \\
S5 & -6 & 1 & 2 \\
S6 & 12 & 0 & 5 \\
\hline
\end{tabular}

Clinical measurements characterizing ankle motor impairments are described for the paretic side of each participant.

Movement error induced by applying a perturbation at the paretic ankle during walking

Applying the perturbation at the ankle induced a deviation towards plantarflexion in the swing phase of walking, as demonstrated for one participant (S4) in Figure 2B. In this subject, a peak torque perturbation of $4.7 \pm 0.6 \mathrm{Nm}$, located at $82.2 \pm 2.2 \%$ of the gait cycle (Figure $2 \mathrm{~A}$ ) produced an ankle deviation of $6.9 \pm 3.5$ degrees at $83.6 \pm 2.0 \%$ of the gait cycle (Figure 2B).

As mentioned under Methods, the amplitude and position of the torque perturbation were adjusted on an individual basis due to the inter-subject differences in the baseline walking pattern. Perturbation characteristics are presented in Table 3. As the goal of the experiment was to walk with a stable torque perturbation for 50 strides (actual 53.2 \pm 8.6 strides), and considering that it took between 0 and 38 strides to adjust peak torque amplitude, participants walked for a varying number of strides while being actually perturbed (range: 50-111 strides).

\section{Ankle dorsiflexor adaptive capacity to a perturbation during walking in persons post-stroke}

In non-disabled persons, a previous study has shown that walking with this perturbation produced an increase in TA activation around toe off [19]. Similar results occurred in 4 of 6 participants post-stroke. In these participants, a significant increase in TA mean amplitude in the swing phase, ranging from $21.0 \%$ to $288.6 \%$ (\% of baseline; S1: $\mathrm{p}<0.001 ; \mathrm{S} 3: \mathrm{p}=0.011 ; \mathrm{S} 4: \mathrm{p}<0.001$; and S6: $p<0.001)$, was observed at the end of the PERTURBATION walking period (Figure $3 \mathrm{~A}$ and $\mathrm{B}$ ). This effect was not present in S2 or S5, however. In S2, the baseline TA activation during swing was so low that the usual stance-to-swing transition burst was undistinguishable from background noise, and changes in TA activation during swing were thus difficult to interpret. In S5, walking with the perturbation did not have a statistically significant impact on TA mean amplitude in the specific zone of the gait cycle $(\mathrm{p}=0.222)$.
Walking with the perturbation did not induce concomitant activation of the antagonist muscle (SOL) during the swing phase in 5 of the 6 participants (Figure $3 \mathrm{C}$ and D; S1: $\mathrm{p}=0.393 ; \mathrm{S} 2: \mathrm{p}=0.056 ; \mathrm{S} 3: \mathrm{p}=0.064 ; \mathrm{S} 4: \mathrm{p}=$ 0.685; S6: $\mathrm{p}=0.432$ ). In the other participant (S5), SOL mean amplitude increased significantly from $28.8 \pm$ $0.8 \mu \mathrm{V}$ at baseline to $35.7 \pm 1.0 \mu \mathrm{V}$ in adapted state, corresponding to an increase of $24.0 \%(p=0.001)$.

Among the 4 participants showing an increased TA activation during the PERTURBATION period, these effects carried over after perturbation removal in 3 of them. Persisting increases in TA mean amplitude in the first strides after removing the perturbation ranged between $24.0 \%$ and $251.5 \%$ (\% of baseline; Figure 3). These aftereffects were significantly different from baseline (S1: $\mathrm{p}<0.001 ; \mathrm{S} 4: \mathrm{p}=0.013 ; \mathrm{S} 6: \mathrm{p}=0.007)$. The relationship between changes in TA activation obtained during and after exposure to the perturbation can be observed in Figure 4. This relationship was nearly linear, as demonstrated by a coefficient of determination value $\left(R^{2}\right)$ of 0.9976. It indicates that more than $99 \%$ of the variance in the modifications in TA amplitude observed after perturbation removal may be explained by the modifications observed with the perturbation.

As mentioned above, the number of strides taken with the perturbation varied across participants (between 50 and 111 strides). However, no relationship was found between exposure duration and aftereffects magnitude (data not shown).

These aftereffects in TA activation led to modifications in ankle angular displacement. Significant increases of $6.6^{\circ}(\mathrm{p}<0.001), 4.2^{\circ}(\mathrm{p}<0.001)$, and $1.9^{\circ}(\mathrm{p}=0.006)$ in maximal dorsiflexion during the swing phase when compared to baseline can be observed after removing the perturbation, in S1, S4, and S6, respectively (Figure 5).

\section{Discussion}

The purpose of the present case series was to evaluate ankle dorsiflexor residual adaptive capacity in persons who had sustained a stroke by having them walk on a treadmill with an electrohydraulic ankle-foot-orthosis that applied a torque perturbation at the ankle joint in the swing phase of the gait cycle.

\section{Walking with the EHO}

It was first necessary to investigate the feasibility of using the EHO to apply a perturbation at the ankle during the swing phase of walking in persons post-stroke. Despite the presence of ankle motor impairments associated with their lesions, participants were able to clear the ground and to walk safely with the EHO. When questioned about their reactions to walking with the EHO, participants reported mild discomfort due to the added mass and the limitation in medio-lateral ankle 


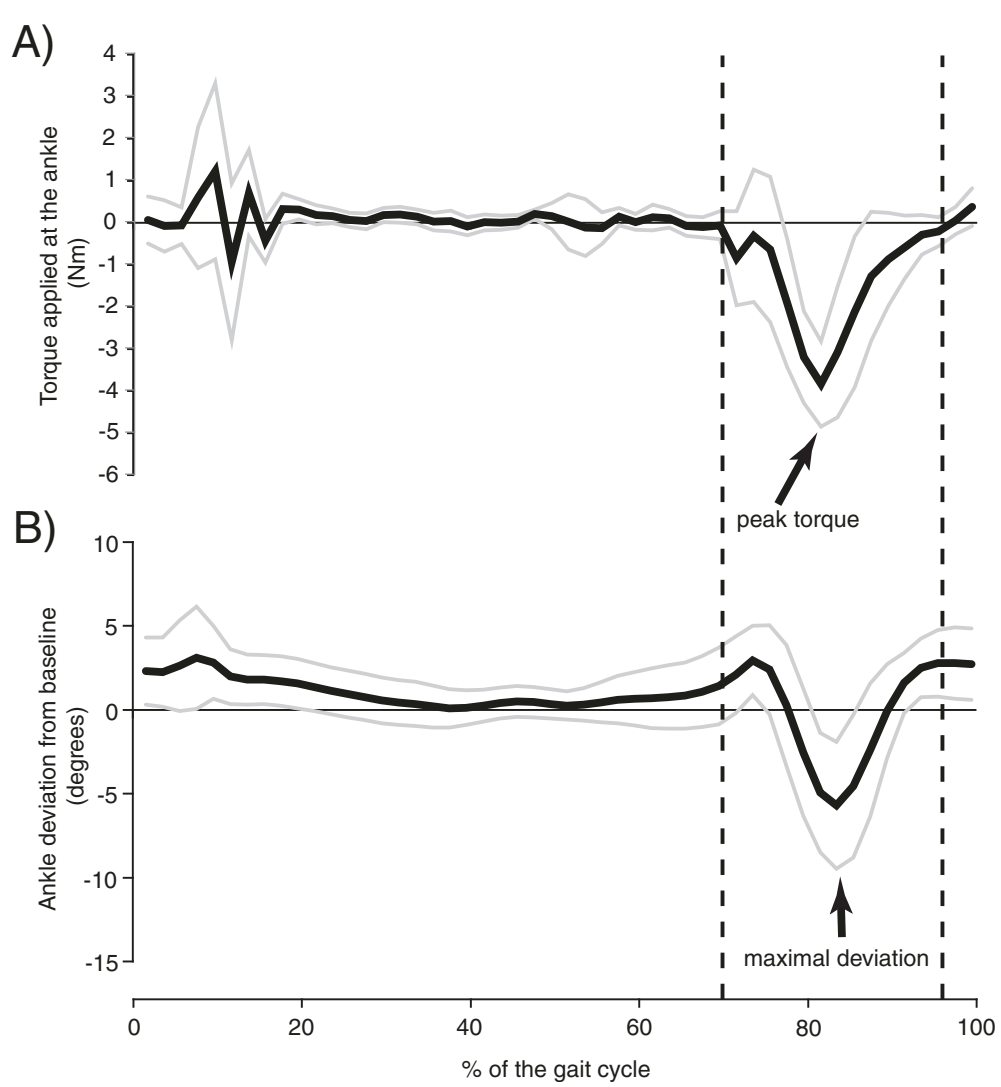

Figure 2 Torque perturbation profile and deviation induced in ankle angular displacements during walking for one participant (S4).

In $\mathbf{A}$, torque applied at the ankle during the walking period with the perturbation for one subject. In $\mathbf{B}$, deviation induced in ankle angular displacements by walking with the perturbation for one subject. Black and grey lines represent the mean and SD $( \pm)$ for all strides with the perturbation, respectively. Vertical dashed lines represent the onset and offset of the torque application.

movements. The aluminum structure was optimized to combine low weight properties with the capacity to sustain sufficient torques. The total mass of the EHO without the shoe is $1.70 \mathrm{~kg}$ [25]. This weight seems reasonable considering that adding a unilateral weight of $1.82 \mathrm{~kg}$ or less does not result in a significant change in walking speed or alter oxygen consumption in non-disabled persons [33]. The

Table 3 Torque perturbation characteristics

\begin{tabular}{cccc}
\hline Participant & $\begin{array}{c}\text { Amplitude } \\
\text { of peak } \\
\text { torque. Nm }\end{array}$ & $\begin{array}{c}\text { Position of peak } \\
\text { torque, \% of the } \\
\text { gait cycle }\end{array}$ & $\begin{array}{c}\text { Maximal plantarflexion } \\
\text { deviation during } \\
\text { swing, degrees }\end{array}$ \\
\hline S1 & $1.9 \pm 0.5$ & $80.1 \pm 2.8$ & $2.4 \pm 3.0$ \\
S2 & $3.0 \pm 0.6$ & $82.8 \pm 2.5$ & $15.6 \pm 3.2$ \\
S3 & $3.2 \pm 0.8$ & $80.8 \pm 2.5$ & $5.6 \pm 3.3$ \\
S4 & $4.7 \pm 0.6$ & $82.2 \pm 2.2$ & $6.9 \pm 3.5$ \\
S5 & $2.5 \pm 0.5$ & $76.5 \pm 2.0$ & $4.2 \pm 1.3$ \\
S6 & $3.0 \pm 0.4$ & $76.2 \pm 4.6$ & $2.1 \pm 1.8$ \\
\hline
\end{tabular}

Torque perturbation application is characterized by the following variables for each participant: amplitude and position of the peak torque, as well as maximal ankle plantarflexion deviation induced by the perturbation $($ mean $\pm S D)$ results of the present study show that persons post-stroke can safely walk with the EHO.

\section{Adaptive capacity to a perturbation during walking in persons post-stroke}

The present study evaluated the dorsiflexor adaptive capacity in 6 persons post-stroke by walking with a perturbation that plantarflexed the ankle during the swing phase. Similar to the results obtained in non-disabled individuals [19], walking with this specific perturbation led to an increased TA activation in 4 of 6 participants, indicating that these individuals still had the capacity to adapt dorsiflexor activation to changes in environmental demands. Furthermore, the modification in TA activation carried over after perturbation removal in 3 of these 4 participants. These results are consistent with a central recalibration of the motor commands (feedforward mechanisms; [34]).

The absence of aftereffects in S3 suggests that modifications in TA activation observed in the presence of the perturbation did not involve a central recalibration of the motor commands. For this particular subject, the 


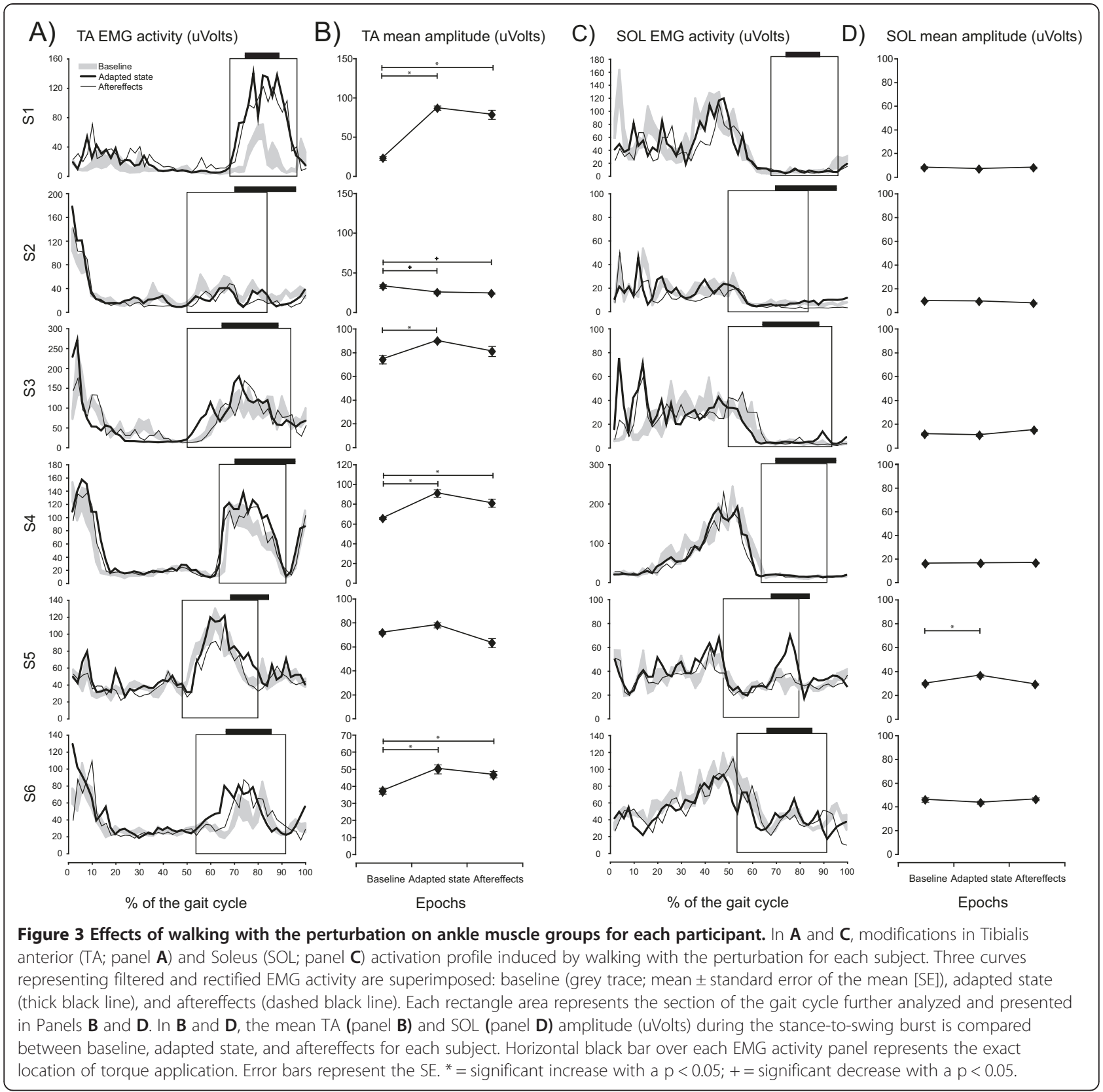

increased TA activation in the adapted state may therefore have been due to modifications in the sensory afferent pattern resulting from the action of the perturbation on the lower limb while walking. By moving the ankle towards plantarflexion during the swing phase, the perturbation may have modified spindle afferent discharge as a result of a different muscle elongation/shortening pattern during walking [35]. Moreover, skin afferent discharge may also have been modified in the presence of the perturbation. When the perturbation was applied, the shoe pushed on the dorsal surface of the foot to move the ankle towards plantarflexion. Considering that feedback mechanisms are involved in the neural control of locomotion $[1,36]$, this modified sensory afferent pattern likely participated to the reshaping of TA muscle activation during walking.

Targeted modifications in TA activation were not obtained in all participants, however. In the remaining 2 participants, no significant increase was observed in TA activation during swing. While S2 did not modify activations of any of the recorded muscles to counteract the effects of the perturbation, S5 increased SOL activation during swing. The resulting TA-SOL coactivation around the ankle joint may have been used to minimize the effects of the perturbation on his walking pattern. The central nervous system is known to adapt the 


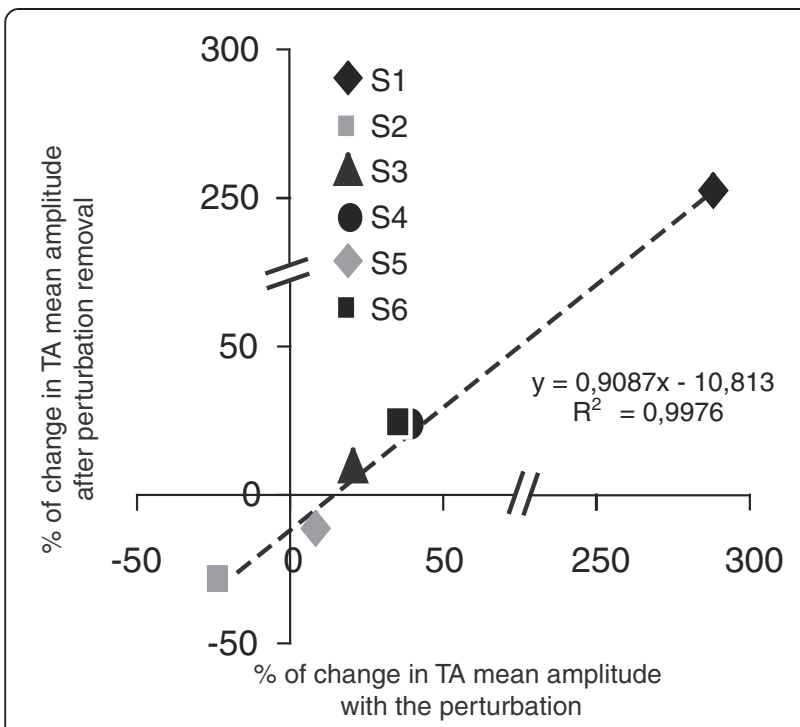

Figure 4 Relationship between changes in TA mean amplitude observed with the perturbation and after its removal. Modifications in TA mean amplitude observed after perturbation removal (aftereffects;\% of baseline) in relation to modifications observed with the perturbation (adapted state; $\%$ of baseline) for each participant. Each symbol represents a single participant. Dashed line consists of a 2-parameter linear fit $\left(R^{2}=0.9976\right)$.

mechanical impedance (stiffness) of the joint by modulating coactivation of antagonist muscle groups during perturbed reaching movements [37-40]. A similar strategy may be adopted in the lower limb when post-stroke persons are walking in a perturbing environment.

Other features of the adaptive capacity in the presence of a perturbation after a stroke have been previously studied $[21,22,24,41]$. Indeed, Reisman et al. [21,22] have demonstrated that persons post-stroke are able to adapt their step length and double support time of the paretic limb to different belt speed relationships (split-belt locomotion). Moreover, modifications in walking symmetry were obtained in a recent study following adaptation to a perturbation that resisted lower limb forward movement with a system of pulleys and weights [24]. These results suggest that, after a cerebral lesion, the impaired CNS is still capable of adapting spatiotemporal gait parameters to a perturbing environment. However, modifications of these inter-limb coordination parameters may involve compensations rather than actual adaptation in some participants. Contrary to these studies, the approach presented here enables the assessment of residual adaptive capacity in a targeted muscle of a single limb during the swing phase, thereby limiting possible contralateral limb compensations. That being said, we are aware that it is very likely that inducing modifications in a muscle group during walking may have consequences on the dynamic control of the whole limb and therefore also affect other muscle groups.

\section{The impact of ankle motor impairments on dorsiflexor adaptive capacity}

Results of the present work also showed that chronic motor impairments affecting the paretic ankle joint are not good predictors of a lack of adaptive capacity in TA muscle activation when walking with the perturbation, as demonstrated by $\mathrm{S} 1$ who presented the most pronounced ankle motor impairments of all participants. In this specific participant, clinical measurements indicated a severe plantarflexor hypertonicity with a marked contracture (ankle passive dorsiflexion did not reach the

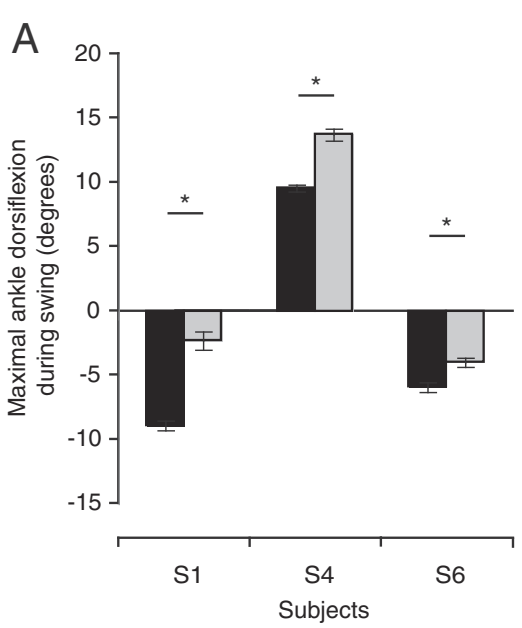

B

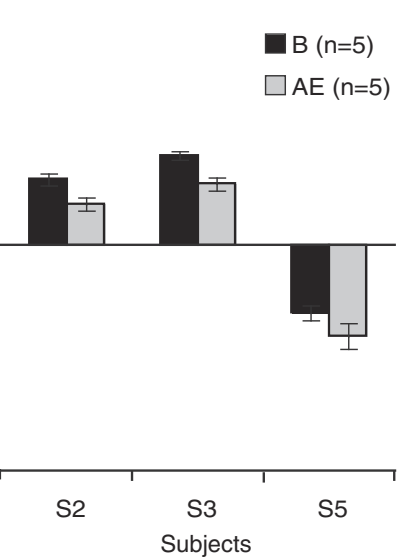

Figure 5 Effects of walking with the perturbation on maximal ankle dorsiflexion during swing after perturbation removal. Maximal ankle dorsiflexion during swing (in degrees) is compared between baseline (B; black bar) and aftereffects (AE; grey bar) for each participant. Panel $\mathbf{A}$ includes the 3 participants showing aftereffects in TA mean amplitude, while Panel $\mathbf{B}$ includes the others. Error bars represent the SE. ${ }^{*}=$ significant increase $(p<0.05)$. 
neutral position), as well as an inability to produce a voluntary dorsiflexion movement. Despite the severity of his ankle motor impairments, S1 demonstrated a very good dorsiflexor adaptive capacity when walking with the perturbation. The lack of correlation between impairments and adaptive plasticity during split-belt locomotion in stroke patients was also reported in Reisman et al. [21]. Further work with larger sample sizes is needed to examine the relationship between specific motor impairments or walking deficits and adaptive capacity.

\section{Clinical implications}

Several authors have suggested that walking in a perturbing environment could be helpful in the rehabilitation of persons with walking limitations following CNS lesions (e.g. [19,21,22,24,42-46]). The premise is that amplifying a walking deficit produces a larger movement error that in turn may trigger a recalibration in central commands to ultimately correct it. As an example, a few protocols voluntarily heighten the asymmetrical locomotor pattern of persons post-stroke by walking with different belt speed relationships (split-belt locomotion; $[21,22])$. Aftereffects resulting from the adaptation to this perturbing environment then improved walking symmetry.

The demonstration that inducing a plantarflexion deviation during walking triggers a central recalibration of the motor commands that leads to an increase in ankle dorsiflexion after perturbation removal supports the potential of such an approach to improve walking limitations. This innovative approach of inducing "angular" movement error, enables the targeting of a specific joint and/or phase-specific motor impairment during walking, such as foot drop (see below) that cannot be addressed with systems like split-belt treadmills.

To date, only a few studies have documented the longterm effects of gait training based on walking in a perturbing environment to improve specific walking disabilities resulting from a cerebral lesion [44,47]. More specifically, multiple sessions of walking with a weight attached on the paretic leg have been reported to lead to improvements in functional ambulation of persons poststroke, particularly in locomotor activities requiring knee and hip flexion, such as stair climbing [47]. Furthermore, a better step length symmetry of a person post-stroke was reported after split-belt treadmill training [44].

Considering that an increase of 5 degrees in dorsiflexion range of movement is usually considered as clinically significant [48], some may argue that statistically significant increases in ankle dorsiflexion obtained in the present study (6.6, 4.2 and 1.9 degrees) might not have a clinical impact on locomotion. However, these effects were induced by a single session of walking with the perturbation (duration ranging between 1 and $3 \mathrm{mi}-$ nutes). It is reasonable to hypothesize that persons presenting a reduced ankle dorsiflexion during the swing phase of walking ("foot drop") may benefit from gait training (multiple sessions) based on this approach. The potential clinical impact of an increased dorsiflexion during the swing phase of walking is likely related to an increase in toe clearance that in turn will facilitate forward body progression and reduce the risk of tripping or falling.

Before testing a locomotor training program based on walking with this specific perturbation on persons poststroke, it is important to address whether the modifications in TA activation transfer to overground walking. To our knowledge, only one study has documented the transfer of aftereffects induced by a perturbation during treadmill walking to overground walking in persons post-stroke [22]. In that study, a partial transfer of the aftereffects induced by walking on a split-belt treadmill to overground walking occurred in non-disabled and post-stroke subjects, suggesting that some aspects of the neural control of overground walking are shared with split-belt treadmill walking. The authors suggest that the transfer would be larger if more characteristics were common to both environmental contexts. Further work is needed to document the transfer of the aftereffects resulting from adaptation to a perturbation such as that used in the present study to overground walking.

\section{Study limitations}

The demonstration of the potential of this approach to evaluate the residual adaptive capacity in the ankle dorsiflexors after a stroke in only six subjects could be considered a study limitation. As demonstrated, however, the evaluation of the adaptive capacity must be made on an individual basis using intra-subject comparative analysis. This therefore greatly reduces the number of subjects needed for a case-series study, like this one.

Sensory impairments were not documented in details in the present study. Only participants with no light touch sensation on the foot or around the ankle were excluded to avoid pressure sores. Considering the contribution of sensory inputs to the control of walking $[1,36]$, it is possible that they would correlate better with adaptive capacity. While results presented in the literature vary with regard to the role of sensory impairments in motor adaptation (see $[21,49]$ ), we suggest that sensory impairments should nevertheless be considered in future studies. Current methods to test proprioceptive deficits at the lower limb are often based on simple tests such as identifying the direction of passive movement of the big toe [21]. As it is reasonable to believe that adaptation while walking with a perturbation may be influenced by the capacity to detect errors in lower limb joint position, 
further work is needed to first develop a valid and reliable proprioception measurement tool for person with motor deficits and then to investigate the relationship between proprioceptive deficits and adaptive capacity.

While no major limitations were found in using the EHO to apply perturbations at the ankle of persons post-stroke during treadmill walking, the need to use customized shoes restricted somewhat our subject selection. Indeed, persons having no light touch sensation on the foot and around the ankle had to be excluded to avoid potential skin damage when the EHO was worn. An improvement would be to modify the design of the EHO to allow personal shoes to be used.

\section{Competing interests}

The authors declare that they have no competing interests.

\section{Authors' contributions}

AKB participated in the design of the study, was responsible for data collection, carried out the data analysis, performed the statistical analysis, and drafted the manuscript. MN was responsible for the software modifications and control of the robotized orthosis during data acquisition, participated in data collection, and helped to draft the manuscript. CLR contributed to the design of the study and helped to draft the manuscript. SN contributed to the design of the study and revised the manuscript. LIB conceived the study, participated in its design, coordination and analysis, and helped to draft the manuscript. All authors read and approved the final manuscript.

\section{Acknowledgements}

The authors would like to thank F. Comeau, D. Tardif and C. Mercier for their technical contribution during data collection, as well as F. Pelletier and the physical therapists (Stroke Unit) of the Quebec Rehabilitation Institute (IRDPQ) for their valuable assistance in participant recruitment. AKB's doctoral studies were supported by the Multidisciplinary Team in Locomotor Rehabilitation (Canadian Institutes of Health Research Team Grant RMF79027) and by the Centre for interdisciplinary research in rehabilitation and social integration (CIRRIS). This work was supported by Multidisciplinary Team in Locomotor Rehabilitation (Canadian Institutes of Health Research Team Grant) and the Fonds de la recherche en santé du Québec.

\section{Author details}

${ }^{1}$ Multidisciplinary Team in Locomotor Rehabilitation, Canadian Institutes of Health Research, Quebec, Canada. ${ }^{2}$ Center for Interdisciplinary Research in Rehabilitation and Social Integration, 525 Wilfrid-Hamel Blvd, Quebec City G1M 2S8, Canada. ${ }^{3}$ Department of Rehabilitation, Université Laval, 1050, ave de la Médecine, room 4472, Quebec City G1V 0A6, Canada. ${ }^{4}$ Centre for Interdisciplinary Research in Rehabilitation of Greater Montreal, 6300 avenue Darlington, Montréal, QC H3S 2 J4, Canada. ${ }^{5}$ School of Rehabilitation, Université de Montreal, Montreal, Canada.

Received: 18 March 2013 Accepted: 2 June 2014

Published: 9 June 2014

\section{References}

1. Nielsen JB, Sinkjaer T: Reflex excitation of muscles during human walking. Adv Exp Med Biol 2002, 508:369-375.

2. Thach WT, Bastian AJ: Role of the cerebellum in the control and adaptation of gait in health and disease. Prog Brain Res 2004, 143:353-366.

3. Barthélemy D, Grey MJ, Nielsen JB, Bouyer L: Involvement of the corticospinal tract in the control of human gait. Prog Brain Res 2011, 192:181-197.

4. Barthélemy D, Alain S, Grey MJ, Nielsen JB, Bouyer L: Rapid changes in corticospinal excitability during force field adaptation of human walking. Exp Brain Res 2012, 217(1):99-115.
5. Dietz V, Zijlstra W, Duysens J: Human neuronal interlimb coordination during split-belt locomotion. Exp Brain Res 1994, 101(3):513-520.

6. Gordon CR, Fletcher WA, Melvill Jones G, Block EW: Adaptive plasticity in the control of locomotor trajectory. Exp Brain Res 1995, 102(3):540-545.

7. Prokop T, Berger W, Zijlstra W, Dietz V: Adaptational and learning processes during human split-belt locomotion: interaction between central mechanisms and afferent input. Exp Brain Res 1995, 106(3):449-456.

8. Emken JL, Reinkensmeyer DJ: Robot-enhanced motor learning: accelerating internal model formation during locomotion by transient dynamic amplification. IEEE Trans Neural Syst Rehabil Eng 2005, 13:33-39.

9. Reisman DS, Block HJ, Bastian AJ: Interlimh coordination during locomotion: what can be adapted and stored? J Neurophysiol 2005, 94:2403-2415.

10. Lam T, Anderschitz M, Dietz V: Contribution of feedback and feedforward strategies to locomotor adaptations. J Neurophysiol 2006, 95(2):766-773.

11. Noble JW, Prentice SD: Adaptation to unilateral change in lower limb mechanical properties during human walking. Exp Brain Res 2006, 169:482-495.

12. Choi JT, Bastian AJ: Adaptation reveals independent control networks for human walking. Nat Neurosci 2007, 10:1055-1062.

13. Gordon KE, Ferris DP: Learning to walk with a robotic ankle exoskeleton. J Biomech 2007, 40(12):2636-2644.

14. Blanchette A, Bouyer $\mathrm{L}$ : Timing-specific transfer of adapted muscle activity after walking in an elastic force field. J Neurophysiol 2009, 102(1):568-577.

15. Fortin K, Blanchette A, McFadyen BJ, Bouyer $\sqcup$ : Effects of walking in a force field for varying durations on aftereffects and on next day performance. Exp Brain Res 2009, 199:145-155.

16. Kao PC, Ferris DP: Motor adaptation during dorsiflexion-assisted walking with a powered orthosis. Gait Posture 2009, 29(2):230-236.

17. Noel M, Fortin $\mathrm{K}$, Bouyer $\mathrm{L}$ : Using an electrohydraulic ankle foot orthosis to study modifications in feedforward control during locomotor adaptation to force fields applied in stance. J Neuroeng Rehabil 2009, 3:6-16.

18. Savin DN, Tseng SC, Morton SM: Bilateral adaptation during locomotion following a unilaterally applied resistance to swing in nondisabled adults. J Neurophysiol 2010, 104(6):3600-3611.

19. Blanchette $A$, Lambert $\mathrm{S}$, Richards $\mathrm{CL}$, Bouyer LJ: Walking while resisting a perturbation: effects on ankle dorsiflexor activation during swing and potential for rehabilitation. Gait Posture 2011, 34(3):358-363.

20. Malone LA, Vasudevan EV, Bastian AJ: Motor adaptation training for faster relearning. J Neurosci 2011, 31:15136-15143.

21. Reisman DS, Wityk R, Silver K, Bastian AJ: Locomotor adaptation on a split-belt treadmill can improve walking symmetry post-stroke. Brain 2007, 130:1861-1872.

22. Reisman DS, Wityk R, Silver K, Bastian AJ: Split-belt treadmill adaptation transfers to overground walking in persons poststroke. Neurorehabil Neural Repair 2009, 23(7):735-744

23. Reisman DS, Bastian AJ, Morton SM: Neurophysiologic and rehabilitation insights from the split-belt and other locomotor adaptation paradigms. Phys Ther 2010, 90:187-195.

24. Savin DN, Tseng SC, Whitall J, Morton SM: Poststroke hemiparesis impairs the rate but not magnitude of adaptation of spatial and temporal locomotor features. Neurorehabil Neural Repair 2012, 27(1):24-34.

25. Noël M, Cantin B, Lambert S, Gosselin CM, Bouyer L: An electrohydraulic actuated ankle foot orthosis to generate force fields and to test proprioceptive reflexes during human walking. IEEE Trans Neural Syst Rehabil Eng 2008, 16(4):390-399.

26. Blanchette $\mathrm{A}$, Richards $\mathrm{CL}$, Bouyer $\mathrm{L}$ : An alternative approach to improve dorsiflexor activation during walking for persons with a central nervous system lesion: a case series [abstract], 16th International WCPT Congress. Amsterdam, The Netherlands: 2011. Abstract Ref. \# RR-PO-207-8-Thu.

27. Perry J: Gait analysis: normal and pathological function. Thorofare, NJ: SLACK Inc.; 1992.

28. Lamontagne A, Richards CL, Malouin F: Coactivation during gait as an adaptive behavior after stroke. J Electromyogr Kinesiol 2000, 10(6):407-415.

29. Moseley AM, Crosbie J, Adams R: Normative data for passive ankle plantarflexion-dorsiflexion flexibility. Clin Biomech (Bristol, Avon) 2001, 16(6):514-521.

30. Bohannon RW, Smith MB: Interrater reliability of a modified Ashworth scale of muscle spasticity. Phys Ther 1987, 67(2):206-207. 
31. Hislop HJ, Montgomery J: Daniels and Worthingham's muscle testing: techniques of manual examination. 7th edition. Philadelphia: W.B. Saunders Company; 2002.

32. Freriks B, Hermens H, Disselhorst-Klug C, Rau G: The recommendations for sensors and sensor placement procedures for surface electromyography, SENIAM 8: European recommendations for surface electromyography. Enschede, The Netherlands: Roessingh Research and Development BV; 1999:15-53.

33. Barnett SL, Bagley AM, Skinner HB: Ankle weight effect on gait: orthotic implications. Orthopedics 1993, 16(10):1127-1131.

34. Lackner JR, DiZio PA: Aspects of body self-calibration. Trends Cogn Sci 2000, 4:279-288.

35. Taylor A, Durbaba R, Ellaway PH, Rawlinson S: Static and dynamic gammamotor output to ankle flexor muscles during locomotion in the decerebrate cat. J Physiol 2006, 571:711-723.

36. Grey MJ, Nielsen JB, Mazzaro N, Sinkjaer T: Positive force feedback in human walking. J Physiol 2007, 581:99-105.

37. Burdet E, Osu R, Franklin DW, Milner TE, Kawato M: The central nervous system stabilizes unstable dynamics by learning optimal impedance. Nature 2001, 414(6862):446-449.

38. Franklin DW, Osu R, Burdet E, Kawato M, Milner TE: Adaptation to stable and unstable dynamics achieved by combined impedance control and inverse dynamics model. J Neurophysiol 2003, 90(5):3270-3282.

39. Gribble PL, Mullin LI, Cothros N, Mattar A: Role of cocontraction in arm movement accuracy. J Neurophysiol 2003, 89(5):2396-2405.

40. Milner TE, Franklin DW: Impedance control and internal model use during the initial stage of adaptation to novel dynamics in humans. J Physiol 2005, 567:651-664.

41. Regnaux JP, Pradon D, Roche N, Robertson J, Bussel B, Dobkin B: Effects of loading the unaffected limb for one session of locomotor training on laboratory measures of gait in stroke. Clin Biomech 2008, 23:762-768.

42. Bastian AJ: Understanding sensorimotor adaptation and learning for rehabilitation. Curr Opin Neurol 2008, 21:628-633.

43. Lam T, Wirz M, Lünenburger L, Dietz V: Swing phase resistance enhances flexor muscle activity during treadmill locomotion in incomplete spinal cord injury. Neurorehabil Neural Repair 2008, 22(5):438-446.

44. Reisman DS, McLean H, Bastian AJ: Split-belt treadmill training poststroke: a case study. J Neurol Phys Ther 2010, 34(4):202-207.

45. Lam T, Pauhl K, Krassioukov A, Eng JJ: Using robot-applied resistance to augment body-weight-supported treadmill training in an individual with incomplete spinal cord injury. Phys Ther 2011, 91(1):143-151.

46. Yen SC, Schmit BD, Landry JM, Roth H, Wu M: Locomotor adaptation to resistance during treadmill training transfers to overground walking in human SCl. Exp Brain Res 2011, 216(3):473-482.

47. Lam T, Luttmann K, Houldin A, Chan C: Treadmill-based locomotor training with leg weights to enhance functional ambulation in people with chronic stroke: a pilot study. J Neurol Phys Ther 2009, 33(3):129-135.

48. Rose KJ, Burns J, Wheeler DM, North KN: Interventions for increasing ankle range of motion in patients with neuromuscular disease. Cochrane Database Syst Rev 2010, 17(2):CD006973.

49. Dukelow SP, Herter TM, Bagg SD, Scott SH: The independence of deficits in position sense and visually guided reaching following stroke. J Neuroeng Rehabil 2012, 9:72

doi:10.1186/1743-0003-11-98

Cite this article as: Blanchette et al:: Modifications in ankle dorsiflexor activation by applying a torque perturbation during walking in persons post-stroke: a case series. Journal of NeuroEngineering and Rehabilitation 2014 11:98

\section{Submit your next manuscript to BioMed Central and take full advantage of:}

- Convenient online submission

- Thorough peer review

- No space constraints or color figure charges

- Immediate publication on acceptance

- Inclusion in PubMed, CAS, Scopus and Google Scholar

- Research which is freely available for redistribution 